\title{
Electron conductivity and second generation Composite Fermions
}

\author{
Matteo Merlo ${ }^{1}$, Nicodemo Magnoli ${ }^{2}$, Maura Sassetti ${ }^{1}$ and Bernhard Kramer ${ }^{3}$ \\ ${ }^{1}$ Dipartimento di Fisica, INFM-LAMIA, Università di Genova, Via Dodecaneso 33, I-16146 Genova, Italy \\ ${ }^{2}$ Dipartimento di Fisica, INFN, Università di Genova, Via Dodecaneso 33, I-16146 Genova, Italy \\ ${ }^{3}$ I. Institut für Theoretische Physik, Universität Hamburg, Jungiusstraße 9, D-20355 Hamburg, Germany
}

(Dated: January 7, 2019)

\begin{abstract}
The relation between the conductivity tensors of Composite Fermions and electrons is extended to second generation Composite Fermions. It is shown that it crucially depends on the coupling matrix for the Chern-Simons gauge field. The results are applied to a model of interacting Composite Fermions that can explain both the anomalous plateaus in spin polarization and the corresponding maxima in the resistivity observed in recent transport experiments.
\end{abstract}

PACS numbers: 71.10.Pm; 73.43.Cd; 73.43.Nq

Keywords: composite fermions; conductivity

Many aspects of the Fractional Quantum Hall Effect $(\mathrm{FQHE})^{1}$ can be understood in terms of the Composite Fermion $(\mathrm{CF})$ model ${ }^{2.3 .4}$ In the field theoretical approach, $\mathrm{CF}$ are constructed by attaching an even number $\tilde{\varphi}$ of flux quanta to an electron via a Chern-Simons (CS) gauge field. In mean field approximation, the Coulomb repulsion between the electrons is removed and the gauge field partly cancels the external vector potential, so that $\mathrm{CF}$ experience a reduced effective magnetic field $\Delta B$ and are otherwise free. For certain values of the electron filling factor $\nu, \Delta B=0$ and $\mathrm{CF}$ form a Fermi sea. For $\Delta B \neq 0, C F$ develop Landau levels. Thus, the principal sequence of FQHE states of electrons at filling factors $\nu=1 / 3,2 / 5, \ldots$ can be viewed as the QHE of noninteracting $\mathrm{CF}$ at integer fillings $p=1,2, \ldots$. This explains many of the observed FQHE states. Fluctuations of the gauge field around the mean value induce an effective interaction between the CF. In the simplest approximation, this can be incorporated into a finite effective mass of the $\mathrm{CF}, m^{*} \propto \sqrt{B} \stackrel{4}{*}$ The quantization of the conductivity of the electrons to the correct fractional values of $e^{2} / h$ is obtained by including in Random Phase Approximation (RPA) the gauge field fluctuations $\underline{\underline{5}}^{-}$

Including the electron spin,,$\frac{6}{}$ the mean field $\mathrm{CF}$ picture can be used to understand the spin polarization of the ground state $\mathrm{I}^{\underline{7}}$ This has been detected in experiments at fixed $\nu$, where the spin polarization $\gamma$ has been measured optically as a function of the magnetic field. It shows crossovers between non-fully polarized states at low magnetic field and completely polarized states at high magnetic field, $\gamma=1 \stackrel{8}{\underline{8}}$ In these experiments, plateaus of intermediate polarization have been detected. These cannot be explained by using the mean field approach. It has been argued that the residual $\mathrm{CF}$ interaction can be attractive, thus leading to an instability towards pairing in spin singlet states that can account for the partly polarized plateaus $\frac{9}{9}$ There are two possibilities, namely particle-particle pairs in analogy to the Cooper pairs in superconductivity, and particle-hole pairs. Chern-Simons theories do indeed play a role in suggesting novel possibilities for ground states, even if quantitative estimates of such suggestions should be subjected to independent testing, e.g. in the form of variational wavefunction calculations.

A doublet Chern-Simons gauge field has been proposed to include spin 10 Obviously, there are several possibilities for flux attachments in this case since different CS fluxes can be associated to electrons with opposite spins. The Lagrangian density for the system of interacting electrons in a magnetic field is $\mathcal{L}=\mathcal{L}_{\mathrm{F}}+\mathcal{L}_{\mathrm{CS}}+\mathcal{L}_{\mathrm{I}}+\mathcal{L}_{\mathrm{Z}}$ where ( $\hbar=c=1$ for the moment)

$$
\begin{gathered}
\mathcal{L}_{\mathrm{F}}(\mathbf{r}, t)=\sum_{s=\uparrow, \downarrow} \psi_{s}^{\dagger}(\mathbf{r}, t)\left\{i \partial_{t}+\mu+e a_{0}^{s}(\mathbf{r}, t)\right. \\
\left.-\frac{1}{2 m}\left[i \nabla+e\left(\mathbf{A}(\mathbf{r})-\mathbf{a}^{s}(\mathbf{r}, t)\right)\right]^{2}\right\} \psi_{s}(\mathbf{r}, t), \\
\mathcal{L}_{\mathrm{CS}}(\mathbf{r}, t)=-\frac{e}{\phi_{0}} \sum_{s, s^{\prime}} \Theta_{s s^{\prime}} a_{0}^{s}(\mathbf{r}, t) \hat{\mathbf{z}} \cdot \nabla \times \mathbf{a}^{s^{\prime}}(\mathbf{r}, t), \\
\mathcal{L}_{\mathrm{I}}(\mathbf{r}, t)=-\frac{1}{2} \sum_{s, s^{\prime}} \int \mathrm{d} \mathbf{r}^{\prime} n_{s}(\mathbf{r}, t) V\left(\mathbf{r}-\mathbf{r}^{\prime}\right) n_{s^{\prime}}\left(\mathbf{r}^{\prime}, t\right) .
\end{gathered}
$$

Here, $\mathcal{L}_{\mathrm{F}}$ represents the kinetic energy of the CF ( $m$ electron band mass, $\mu$ chemical potential), $\mathcal{L}_{\mathrm{CS}}$ the ChernSimons doublet field $\left(\phi_{0}=h c / e\right.$ flux quantum, $\hat{\mathbf{z}}$ unit vector perpendicular to the $2 \mathrm{D}$ system), with matrix-valued coupling strength $\Theta_{s s^{\prime}}, \mathcal{L}_{\mathrm{I}}$ the interaction, and $\mathcal{L}_{\mathrm{Z}}$ the Zeeman energy. Furthermore, $n_{s}(\mathbf{r}, t) \equiv \psi_{s}^{\dagger}(\mathbf{r}, t) \psi_{s}(\mathbf{r}, t)$ is the density of the Fermions with spin orientation $s, \mathbf{A}$ the vector potential of the external magnetic field, $\left(a_{0}, \mathbf{a}\right)$ the CS gauge field, and $V(\mathbf{r})=e^{2} / \epsilon r$ the Coulomb interaction potential ( $\epsilon$ host material dielectric constant).

The attachment of flux quanta is achieved by the CSterm as can be seen by minimizing $\mathcal{L}$ with respect to $a_{0}^{s}$,

$$
\sum_{s^{\prime}} \Theta_{s s^{\prime}} \hat{\mathbf{z}} \cdot \nabla \times \mathbf{a}^{s^{\prime}}(\mathbf{r}, t)=\phi_{0} n_{s}(\mathbf{r}, t)
$$

with the symmetric $2 \times 2$ coupling matrix

$$
\Theta=\left(\begin{array}{cc}
\theta_{1} & \theta_{2} \\
\theta_{2} & \theta_{1}
\end{array}\right)
$$

A given CF "sees" a CF with like $\backslash$ unlike spin as carrying a number $\theta_{1 \backslash 2} /\left(\theta_{1}^{2}-\theta_{2}^{2}\right)$ of flux quanta. 
Different choices for $\Theta$ map to CF systems with different properties. This is obvious in the diagonal basis $\mathbf{a}^{ \pm}=1 / 2\left(\mathbf{a}^{\uparrow} \pm \mathbf{a}^{\downarrow}\right)$, in which Eq. (4) reads

$$
\begin{aligned}
\phi_{0}\left[n_{\uparrow}(\mathbf{r}, t)+n_{\downarrow}(\mathbf{r}, t)\right] & =\theta_{+} \hat{z} \cdot \nabla \times \mathbf{a}^{+} \\
\phi_{0}\left[n_{\uparrow}(\mathbf{r}, t)-n_{\downarrow}(\mathbf{r}, t)\right] & =\theta_{-} \hat{z} \cdot \nabla \times \mathbf{a}^{-},
\end{aligned}
$$

with $\theta_{ \pm}=2\left(\theta_{1} \pm \theta_{2}\right)$. For instance, the singular choice $\theta_{-}=0$ demands that locally $n_{\uparrow}(\mathbf{r}, t)=n_{\downarrow}(\mathbf{r}, t)$ and therefore describes a spinless boson of charge $2 e$.

The attachment of the same flux to all electrons independently of the spin can be obtained with $\theta_{-} \rightarrow \infty$ and $\theta_{+}$finite. This decouples $\mathbf{a}^{-}$and leads to a single CS-field $\mathbf{a}^{+}$coupled to all CF. This is the conventional method for describing partly polarized states 10.11 It maps electrons with spin $s$ at filling factor $\nu$ to composite particles consisting of one electron with spin $s$ and $\tilde{\varphi}=\theta_{+}^{-1}$ flux quanta in an effective field $\Delta B^{s}=$ $\Delta B=B-n(\mathbf{r}) \tilde{\varphi} \phi_{0}$ that is independent of the spin, with $n(\mathbf{r}, t)=n_{\uparrow}(\mathbf{r}, t)+n_{\downarrow}(\mathbf{r}, t)$ the total density and $\tilde{\varphi}$ even to ensure fermionic statistics.

At mean field level, $n(\mathbf{r})$ is replaced by its average $n_{e}, \Delta B$ is uniform and induces quantization into $\mathrm{CF}$ Landau levels (CFLL). One can define CF filling factors $p_{s}=\phi_{0} n_{s} / \Delta B$ that can be related to $\nu$. This gives the principal sequence $\nu=\left(p_{\uparrow}+p_{\downarrow}\right) /\left[\tilde{\varphi}\left(p_{\uparrow}+p_{\downarrow}\right) \pm 1\right]$ with $p_{\uparrow}, p_{\downarrow}$ integer numbers of filled spin up/down CFLL. The limit $\Delta B=0$ corresponds to a Fermi sea at $\nu=1 / \tilde{\varphi}$ and is obtained for $p=p_{\uparrow}+p_{\downarrow} \rightarrow \infty$.

The cyclotron energies $\hbar \Delta \omega_{c}=\hbar e \Delta B / m c$ for both spins scale incorrectly with the magnetic field at fixed filling $p$. Dimensional analysis 4 and explicit calculations ${ }^{12.13}$ yield a renormalized $\hbar \Delta \omega_{c}^{*} \propto E_{\mathrm{C}} /(\tilde{\varphi} p \pm 1)$, with the Coulomb energy $E_{\mathrm{C}}=e^{2} / \epsilon \ell$ and $2 \pi B \ell^{2}=\phi_{0}$ defines the magnetic length $\ell$. This can be recovered in mean field approximation by replacing $m$ with an effective mass $m^{*}=\alpha m_{0} \sqrt{B[\text { Tesla }]}$, with a sample dependent parameter $\alpha$ and $m_{0}$ the electron mass 14 For $\tilde{\varphi}=2$, relevant for filling factors $1 / 3<\nu<1$, one finds $\hbar \Delta \omega_{c}^{*}=\hbar e \sqrt{B} /\left[m_{0} c \alpha(2 p \pm 1)\right]$.

Combining the Landau quantization with the Zeeman term yields spin-split CF Landau levels $\left(\mu_{\mathrm{B}}=\hbar e / 2 m_{0} c\right)$

$$
E_{n p s}(B)=\left(n+\frac{1}{2}\right) \hbar \Delta \omega_{c}^{*}+\frac{1}{2} s g \mu_{\mathrm{B}} B,
$$

with $s=\uparrow, \downarrow= \pm 1$ and the CF Landé factor $g$.

Various properties of the FQHE states at $\nu=p /(2 p \pm 1)$ can be determined using this spectrum and the ground state obtained by filling the lowest $p$ CFLL. For instance, the main crossovers in the spin polarization

$$
\gamma=\frac{n_{\uparrow}-n_{\downarrow}}{n_{\uparrow}+n_{\downarrow}}=\frac{p_{\uparrow}-p_{\downarrow}}{p_{\uparrow}+p_{\downarrow}}
$$

at fixed $\nu$ can be associated with intersections between CFLL with opposite spins at the Fermi energy (Fig 1).

The parameter $\alpha$ and the $\mathrm{CF} g$-factor have been determined by fitting Eq. (7) to measurements of the crossover field $B_{\mathrm{C}}{ }^{7}$ and the activation gap $\frac{15}{2}$ This yields a step-like polarization. However, the experimentally detected halfpolarized plateaus cannot be reproduced.

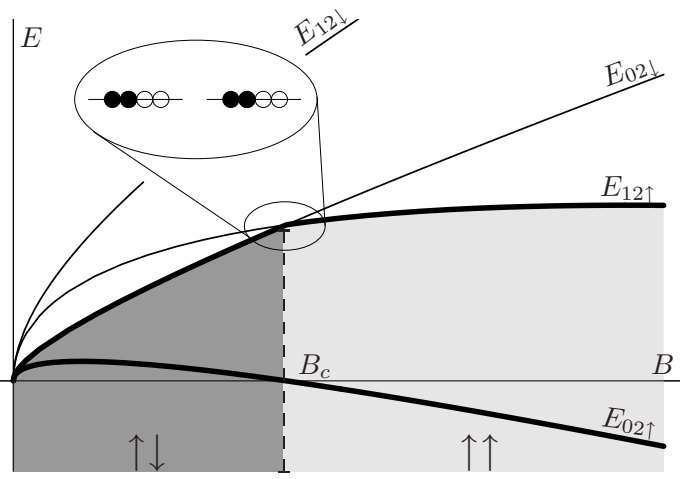

FIG. 1: CFLL for $p=2(\nu=2 / 5)$. The four lowest $E_{n p s}$ of Eq. (7) are shown with arbitrary scales for $E$ and $B$. Bold: lowest $p$ filled CFLL that constitute the ground state; dark grey: $p_{\uparrow}=p_{\downarrow}=1, \gamma=0$; light grey: $p_{\uparrow}=2, p_{\downarrow}=0, \gamma=1$. At the crossover field $B_{c}$, two levels intersect.

Close to $B_{c}$ (Fig 1), $E_{02 \downarrow}$ and $E_{12 \uparrow}$ are almost degenerate and the residual $\mathrm{CF}$ interaction is expected to become relevant 16 We have studied this case by assuming that exactly at degeneracy both levels are half-filled. We have performed another Chern-Simons transformation to create second-generation $\mathrm{CF}\left({ }^{2} \mathrm{CF}\right)$. The lowest CFLL $E_{02 \uparrow}$ is not involved in the level crossing. As before, a suitable choice of the coupling matrix $\Theta$ is necessary to specify the transformation. In this case, it appears natural to perform the flux attachment in an independent way, namely assuming diagonal coupling $\Theta=\operatorname{diag}(\theta)$. While this choice is perfectly legitimate, it differs from the first CS transformation in that both gauge fields $\mathbf{a}^{ \pm}$survive and have to be considered separately. This will turn to be crucial when evaluating the conductivity.

The degenerate system at $B_{c}$ is described by a Lagrangian equivalent to the one above but without the Zeeman term. The constraint Eq. (4) is now $\theta \hat{\mathbf{z}} \cdot \nabla \times$ $\mathbf{a}^{s}(\mathbf{r}, t)=\phi_{0} n_{s}(\mathbf{r}, t)$ where in mean field approximation $n_{\uparrow}=n_{\downarrow}$ because of the assumption of half-filled levels. Each species of CF therefore couples separately to the same-spin CS field. The choice $\theta=1 / 2$ preserves fermionic statistics and maps the system on two Fermi seas at $B=0$ that are coupled by the interaction.

An effective interaction between ${ }^{2} \mathrm{CF}$

$$
\begin{aligned}
& V^{s, s^{\prime}}\left(\mathbf{k}, \mathbf{k}^{\prime}, \mathbf{q} ; \Omega\right)=v^{s}(\mathbf{k}, \mathbf{q}) v^{s^{\prime}}\left(\mathbf{k}^{\prime},-\mathbf{q}\right) \\
& \quad \times\left[\mathcal{D}^{+}(\mathbf{q}, \Omega)+\left(2 \delta_{s s^{\prime}}-1\right) \mathcal{D}^{-}(\mathbf{q}, \Omega)\right]
\end{aligned}
$$

is then obtained considering scattering of states with spin $s$, $s^{\prime}$ and momenta $\mathbf{k}$, and $\mathbf{k}^{\prime}$ into states with the same spins and momenta $(\mathbf{k}+\mathbf{q}),\left(\mathbf{k}^{\prime}-\mathbf{q}\right)$, mediated by the exchange of a gauge field fluctuation with momentum $\mathbf{q}$ and frequency $\Omega$. In Eq. (9), $\mathcal{D}^{ \pm}$are RPA gauge field propagators associated with the symmetric and antisymmetric 
combinations of spin and $v^{s}$ are interaction vertices between ${ }^{2} \mathrm{CF}$ and the CS-field fluctuations. The potential $V^{s, s^{\prime}}$ is attractive in the $s$-wave channel both for particleparticle (p-p) and particle-hole (p-h) pairs with zero total spin. New collective ground states $\left|\Phi_{p p}\right\rangle,\left|\Phi_{p h}\right\rangle$ form, characterized by pair-breaking gaps $\frac{17}{17}$

$$
\begin{aligned}
& \Delta_{p p} \propto\left\langle\Phi_{p p}\left|\psi_{\uparrow}^{\dagger} \psi_{\downarrow}^{\dagger}\right| \Phi_{p p}\right\rangle \\
& \Delta_{p h} \propto\left\langle\Phi_{p h}\left|\psi_{\uparrow}^{\dagger} \psi_{\downarrow}\right| \Phi_{p h}\right\rangle .
\end{aligned}
$$

The energies of the paired ground states depend differently on the strength and range of the interaction so that crossovers between the p-p and the p-h phases can occur 18 Both states are rigid zero-polarization states. Flipping a spin requires a finite amount of energy given by $\Delta_{p p}$ or $\Delta_{p h}$, both of the order $0.5 \mathrm{~K}$ for typical experimental conditions. This can explain the half-polarized plateaus observed experimentally ${ }^{8}$ as signature of the condensation into a paired singlet state which does not contribute towards spin polarization. The condition for this is that the energy gain by forming the paired ground state should be larger than the energy required for exciting the $\mathrm{CF}$ to the higher level. In the example of Fig. 10 the crossing levels $E_{02 \downarrow}$ and $E_{12 \uparrow}$ contribute spin 0, while $E_{02 \uparrow}$ has all spins up. Since the degeneracy is the same for all levels, the total polarization is $\gamma=1 / 2$. This can be generalized to other filling factors, such as $3 / 7$ or $4 / 9$, where more intersections occur, and one can systematically reproduce all observed partially polarized plateaus.

The p-p and p-h pairs binding energy are of the same order, though. Therefore, it is not possible to distinguish between the two phases by comparing the predicted plateau widths with experiment.

To find differences in the nature of the paired states, we consider electrical transport. Since a CF contains charge and flux, a current $\mathbf{j}$ of CF across a closed loop corresponds to a variation of magnetic flux. This yields a CSelectric field $\mathbf{E}^{\mathrm{CS}}$ via Faraday's law $\mathbf{E}^{\mathrm{CS}}=\left(\tilde{\varphi} \phi_{0} / e c\right) \hat{\mathbf{z}} \times \mathbf{j}$ where $\hat{\mathbf{z}}$ is the unit vector perpendicular to the $2 \mathrm{D}$ electron system in the $(x, y)$-plane. Self-consistency requires that $\mathrm{CF}$ respond to both $\mathbf{E}^{\mathrm{CS}}$ and the electric field $\mathbf{E}, \frac{5}{\underline{5}}$

$$
\mathbf{j}=\hat{\sigma}^{\mathrm{CF}}\left(\mathbf{E}+\mathbf{E}^{\mathrm{CS}}\right)
$$

with the CF conductivity $\hat{\sigma}^{\mathrm{CF}}$. Defining CS- and electron resistivities $\mathbf{E}^{\mathrm{CS}}=-\hat{\rho}^{\mathrm{CS}} \mathbf{j}$ and $\mathbf{E}=\hat{\rho} \mathbf{j}$, respectively,

$$
\hat{\rho}=\hat{\rho}^{\mathrm{CF}}+\hat{\rho}^{\mathrm{CS}}
$$

with

$$
\hat{\rho}^{\mathrm{CS}}=\tilde{\varphi} \frac{h}{e^{2}}\left(\begin{array}{cc}
0 & 1 \\
-1 & 0
\end{array}\right)
$$

and $\hat{\rho}^{\mathrm{CF}}=\left[\sigma^{\hat{\mathrm{CF}}}\right]^{-1}$. Equation (13) can be more formally justified using linear response theory in terms of current-current correlation functions $\stackrel{\underline{4}}{\underline{\underline{4}}}$ It has been used successfully for both FQHE and even-denominator QHE states. Its applicability depends on the choice of the coupling matrix $\Theta$. It is correct when a single CS-field couples with the total density, as for spinless fermions. It also holds for the above first CS-transformation with the special choice $\theta_{-} \rightarrow \infty$, finite $\theta_{+}$, where again a single CS-field, in this case $\mathbf{a}^{+}$, couples to the fermions $\frac{19}{19}$

Regarding the situation near the crossover points, we need to determine $\rho_{\mathrm{CF}}$. We split the conductivity of the $p$ levels of $\mathrm{CF}$ into a contribution $\hat{\sigma}_{\mathrm{h}}^{\mathrm{CF}}$ from the highest level, which changes and determines the spin transition, and a contribution from the lowest $p-1$ levels $\hat{\sigma}_{p-1}^{\mathrm{CF}}=\hat{\sigma}^{\mathrm{CF}}-\hat{\sigma}_{\mathrm{h}}^{\mathrm{CF}}$. For levels that are not involved in crossings, $\mathrm{CF}$ are treated as non-interacting fermions in an effective magnetic field. Thus, $\hat{\sigma}_{p-1}^{\mathrm{CF}}$ is the sum of the conductivities $\hat{\sigma}^{\mathrm{LL}}$ of the filled Landau levels,

$$
\hat{\sigma}_{p-1}^{\mathrm{CF}}=(p-1) \hat{\sigma}^{\mathrm{LL}} \equiv(p-1) \frac{e^{2}}{h}\left(\begin{array}{cc}
0 & -1 \\
1 & 0
\end{array}\right) .
$$

The contribution of the highest level depends on whether we are at a spin transition or not. It is again that of a filled CFLL $\hat{\sigma}_{\mathrm{h}}^{\mathrm{CF}}=\hat{\sigma}^{\mathrm{LL}}$ for $B \neq B_{c}$. In this case, the resistivity of quantum Hall states is reproduced,

$$
\begin{aligned}
& \rho_{x x}(B)=0 \\
& \rho_{x y}(B)=\frac{h}{e^{2}} \frac{2 p+1}{p}=\frac{h}{\nu e^{2}} .
\end{aligned}
$$

At $B \approx B_{c}$, the highest occupied level consists of two crossing levels, both half-filled, on which a CS-mapping to ${ }^{2} \mathrm{CF}$ is done. The contribution to transport will then depend on the nature of the state formed at the transition. It appears natural to consider $\hat{\sigma}_{\mathrm{h}}^{\mathrm{CF}}$ using an expression equivalent to Eq. (13) but applied to ${ }^{2} \mathrm{CF}$ and consistent with our assumptions for $\Theta$. Considering the Lagrangian for the two transformed intersecting levels, the corresponding current density will have contributions from both spin up/down ${ }^{2} \mathrm{CF}$ according to $\mathbf{j}=\mathbf{j}_{\uparrow}+\mathbf{j}_{\downarrow}$. Each will induce a fictitious CS electric field felt only by the fermions with equal spins,

$$
\mathbf{E}_{s}^{\mathrm{CS}}=-\hat{\rho}^{\mathrm{CS}} \mathbf{j}_{s},
$$

where the CS-resistivity is given by Eq. (14) because it depends on the number of attached fluxes $(=2)$ in both cases. Linear response gives

$$
\mathbf{j}_{s}=\sum_{s^{\prime}} \hat{\sigma}_{s s^{\prime}}^{2} \mathrm{~F}\left(\mathbf{E}+\mathbf{E}_{s^{\prime}}^{\mathrm{CS}}\right),
$$

with $\hat{\sigma}_{\uparrow \uparrow}^{2 \mathrm{CF}}=\hat{\sigma}_{\downarrow \downarrow}^{2 \mathrm{CF}}$ and $\hat{\sigma}_{\uparrow \downarrow}^{2 \mathrm{CF}}=\hat{\sigma}_{\downarrow \uparrow}^{2 \mathrm{CF}}$ because of spin up/down symmetry. Inserting Eq. (17), one gets

$$
\mathbf{j}=\hat{\sigma}_{h}^{\mathrm{CF}} \mathbf{E}=2\left(\hat{\sigma}_{\uparrow \uparrow}^{2 \mathrm{CF}}+\hat{\sigma}_{\uparrow \downarrow}^{2} \mathrm{CF}\right) \mathbf{E}-\left(\hat{\sigma}_{\uparrow \uparrow}^{2} \mathrm{CF}+\hat{\sigma}_{\uparrow \downarrow}^{2} \mathrm{CF}\right) \hat{\rho}^{\mathrm{CS}} \mathbf{j},
$$

so that the equivalent of Eq. (13) becomes

$$
\hat{\rho}_{h}^{\mathrm{CF}}=\hat{\rho}^{2} \mathrm{CF}+\frac{1}{2} \hat{\rho}^{\mathrm{CS}},
$$


where $\hat{\rho}^{2} \mathrm{CF}=\left[2\left(\hat{\sigma}_{\uparrow \uparrow}^{2} \mathrm{CF}+\hat{\sigma}_{\uparrow \downarrow}^{2} \mathrm{CF}\right)\right]^{-1}$, and $\hat{\rho}_{h}^{\mathrm{CF}}=\left[\hat{\sigma}_{\mathrm{h}}^{\mathrm{CF}}\right]^{-1}$ is the resistivity of the degenerate levels of ${ }^{1} \mathrm{CF}$.

We now calculate the resistivity of the ${ }^{2} \mathrm{CF}$. For noninteracting ${ }^{2} \mathrm{CF}$ in unperturbed Fermi seas, one expects

$$
\hat{\rho}^{2} \mathrm{CF}=\frac{h}{e^{2}}\left(\begin{array}{cc}
\rho_{0} & 0 \\
0 & \rho_{0}
\end{array}\right),
$$

with $\rho_{0}$ the resistance due to impurity scattering.

The $\mathrm{p}-\mathrm{h}$ pairing leads to a modification of the Fermi surfaces that leaves unchanged the response of the system in the presence of impurities. The resistivity $\hat{\rho}_{p h}^{2} \mathrm{CF}$ is given by Eq. (21). On the other hand, since p-p pairing leads to an instability of the CF Fermi sphere in analogy to the BCS theory of superconductivity, the diagonal elements of the resistivity vanish, even with disorder.

Using Eq. (20) we then have

$$
\begin{aligned}
\left.\hat{\sigma}_{\mathrm{h}}^{\mathrm{CF}}\right|_{p h} & =\frac{e^{2}}{h} \frac{1}{\rho_{0}^{2}+1}\left(\begin{array}{cc}
\rho_{0} & -1 \\
1 & \rho_{0}
\end{array}\right) \\
\left.\hat{\sigma}_{\mathrm{h}}^{\mathrm{CF}}\right|_{p p} & =\frac{e^{2}}{h}\left(\begin{array}{cc}
0 & -1 \\
1 & 0
\end{array}\right) .
\end{aligned}
$$

Inserting $\left.\hat{\sigma}_{\mathrm{h}}^{\mathrm{CF}}\right|_{p h}$ into (13), one finds for the $\mathrm{p}$-h phase

$$
\begin{aligned}
\rho_{x x}\left(B_{c}\right) & =\frac{h}{e^{2}} \frac{\rho_{0}}{p^{2}+(p-1)^{2} \rho_{0}^{2}} \\
\rho_{x y}\left(B_{c}\right) & =\frac{h}{e^{2}}\left[\frac{2 p+1}{p}+\frac{\rho_{0}^{2}(p-1)}{p^{3}+p(p-1)^{2} \rho_{0}^{2}}\right] .
\end{aligned}
$$

The result for the p-p state is obtained with $\rho_{0}=0$ which reproduces Eq. (16). Thus, the two states can be distin- guished measuring the resistance. For the $\mathrm{p}$-h state $\rho_{x x}$ and $\rho_{x y}$ will deviate from the ideal quantum Hall values.

Recently, non-universal behavior in $\rho_{x x}$ and $\rho_{x y}$ has been observed 20 near the spin transition, accompanied by hysteresis induced by the coupling between the electron system and the nuclear spins. Previously, this was attributed to ferromagnetic domains in the quantum Hall system which can produce deviations in the resistance components from their ideal values due to scattering at the domain walls. If the above $\mathrm{p}$-h state was formed, this could also account for both the half-polarized spin plateaus and the anomalous behavior of the resistance.

In conclusion, we have extended the standard procedure to relate the conductivities of $\mathrm{CF}$ and electrons to include multiple flux attachment. This is important for understanding the behavior of quantum Hall systems at filling factors where transitions between ground states with different spins occur. In the presence of interactions between $\mathrm{CF}$, we have found that for $\mathrm{p}$ - $\mathrm{h}$ pairing at the spin transition the resistance indicates a breakdown of the quantum Hall phase. If p-p (Cooper) pairs are formed the resistance is not affected. Finally, we would like to mention that experiments suggest similar features to occur at other fillings. For instance, if threequarters/one-quarter filling of CF spin-up/down levels, respectively, was forming a stable correlated many body state, this could account for the three-quarter polarized state observed recently at electron filling $\nu=2 / 3^{21}$

Acknowledgments: Financial support from the EU via MCRTN-CT2003-504574 and from the DFG via Project $\mathrm{Kr} 627 / 12$ of the special research program "Quanten-Hall-Effekt" is gratefully acknowledged.
1 D. C. Tsui, H. L. Stormer and A. C. Gossard, Phys. Rev. Lett. 48, 1559 (1982).

2 J. K. Jain, Phys. Rev. Lett. 63, 199 (1989).

3 A. Lopez and E. Fradkin, Phys. Rev. B 44, 5246 (1991).

4 B. I. Halperin, P. A. Lee, and N. Read, Phys. Rev. B 47, 7312 (1993)

5 S. H. Simon, in Composite Fermions, edited by O. Heinonen (World Scientific, Singapore, 1998).

${ }^{6}$ B. I. Halperin, Helv. Phys. Acta 56, 75 (1983).

7 E. Mariani, R. Mazzarello, M. Sassetti, and B. Kramer, Ann. Phys. (Leipzig) 11, 926 (2002).

8 I. V. Kukushkin, K. von Klitzing, and K. Eberl, Phys. Rev. Lett. 82, 3665 (1999).

9 E. Mariani, N. Magnoli, F. Napoli, M. Sassetti, and B. Kramer, Phys. Rev. B 66, 241303 (2002).

10 S.S. Mandal, V. Ravishankar, Phys. Rev. B 54, 8688 (1996).

11 A. Lopez and E. Fradkin, Phys. Rev. B 51, 4347 (1995).

12 N. d'Ambrumenil and R. Morf, Phys. Rev. B 40, 6108 (1989)

13 A. Stern and B. I. Halperin, Phys. Rev. B 52, 5890 (1995).

14 K. Park and J. K. Jain, Phys. Rev. Lett. 80, 4237 (1998).
15 F. Schulze-Wischeler, E. Mariani, F. Hohls, and R. J. Haug, Phys. Rev. Lett. 92, 156401 (2004).

16 G. Murthy, Phys. Rev. Lett. 84, 350 (2000); V. M. Apalkov, T. Chakraborty, P. Pietilainen, and K. Niemela, Phys. Rev. Lett. 86, 1311 (2001).

17 Despite the name particle-hole suggests a charge neutral object, we want to emphasize that the collective ground state $\Phi_{p h}$ corresponds to a hybridization of the degenerate spin bands and is indeed charged (T. Ando, A.B. Fowler, F. Stern, Rev. Mod. Phys. 54, 437 (1984)).

18 M. Merlo, N. Magnoli, M. Sassetti, and B. Kramer, Phys. Rev. B 71, 155307 (2005).

19 S.S. Mandal, V. Ravishankar, Phys. Rev. B 57, 12333 (1998).

20 J.H. Smet, R. A. Deutschmann, W. Wegscheider, G. Abstreiter, and K. von Klitzing, Phys. Rev. Lett. 86, 2412 (2001).

21 N. Freytag, Y. Tokunaga, M. Horvatic, C. Berthier, M. Shayegan, and L.P. Levy, Phys. Rev. Lett. 87, 136801 (2001). 